\title{
Différentes approches pour la formulation des bétons autoplaçants
}

\section{Influence sur les caractéristiques rhéologiques et mécaniques}

\section{Philippe Turcry**** Ahmed Loukili*}

\author{
* R\&DO, Laboratoire de Génie Civil de Nantes Saint Nazaire \\ Ecole Centrale de Nantes, 1 rue de la Nö̈ \\ BP 92101 F-44321 Nantes cedex 3
}

**VM Matériaux, Laboratoire Qualité

Route de La Roche-sur-Yon, F-85260 L'Herbergement

philippe.turcry@ec-nantes.fr

\begin{abstract}
RÉSUMÉ. La formulation des bétons autoplaçants se fait, dans la plupart des cas, de manière empirique, alors qu'il existe des approches plus rationnelles. On présente ici une comparaison de trois méthodes de formulation récurrentes dans la littérature. La première est une méthode à la fois forfaitaire, pour le dosage des granulats, et expérimentale, pour le dosage en eau et en adjuvant. La deuxième approche propose deux critères pour minimiser le volume de pâte. La troisième approche est basée sur l'optimisation du squelette du béton; il s'agit d'un logiciel compilant un modèle d'empilement granulaire et des modèles rhéologiques. Dans le cadre de la norme P18-305, différents BAP, sans agent de viscosité, ont été formulés avec ces méthodes, puis caractérisés à l'aide des essais préconisés par l'AFGC. Cette étude de faisabilité permet de dresser différents niveaux pour une formulation scientifique des BAP ; elle permet également d'envisager de nouvelles possibilités pour le formulateur. Une étude de l'incidence de la formulation sur les propriétés à l'état durci a également été menée par comparaison avec un béton ordinaire.
\end{abstract}

ABSTRACT. No general method is available in France to optimise SCC composition, and therefore design requires a lot of trials in laboratory or in most of cases directly in plant. However more rational approaches can be found in recent publications. The aim of this paper is to compare three of the most recurrent methods. The first one is a very simple mix design: coarse and fine aggregates contents have fixed values, and superplasticizer and water proportions are determined by mortar tests. The second method proposes two criteria to minimise paste volume. The third approach is based on the packing and on several models describing the properties of the fresh SCC. According to the French Standard P18-305, different SCC have been designed, without viscosity agents, by these methods, and their properties at the fresh state have been characterised with tests advised by the AFGC. The experimental results provide new solutions for SCC designers in an industrial context. A study of the effect of mix design on harden state properties is provided too.

MOTS-CLÉS : bétons autoplaçants, formulation, état frais, état durci.

KEYWORDS: self-compacting concrete, mix design, fresh state properties, hard state properties. 


\section{Introduction}

\subsection{Contexte}

Les fabricants français de bétons se sont lancés ces dernières années dans la vente de nouveaux bétons, caractérisés par une mise en place sans vibration, les bétons autoplaçants (BAP). Inspirés par le concept japonais du béton fluide, leur développement a été initié par les fabricants d'adjuvants qui proposaient à l'époque une nouvelle génération de superplastifiants à fort pouvoir défloculant. Si la production reste minime ( $1 \%$ à $4 \%$ du volume total), leur utilisation n'est pas uniquement limitée aux grands ouvrages. De fait, ils séduisent de plus en plus l'industrie du bâtiment, qui y voit un moyen de supprimer les problèmes, techniques et parfois environnementaux, liés à la vibration.

Les bétons autoplaçants sont toutefois encore marginaux. Leur composition est en effet beaucoup plus complexe que celle des bétons dits ordinaires (BO), dont ils se distinguent par leurs propriétés à l'état frais. Formuler un BAP, c'est trouver l'arrangement optimal - souvent du point de vue économique - entre au moins six constituants différents (gravillons, sables, ciment, additions, eau, adjuvants) permettant l'obtention des propriétés suivantes : grande fluidité, capacité à s'écouler en milieu confiné sans blocage, et caractère autonivelant. Il s'agit également de garantir l'absence de ségrégation et de ressuage. La plupart des formules de BAP sont conçues actuellement de manière empirique ; les méthodes classiques ne sont pas en effet adaptées à la formulation d'un béton fluide contenant additions et adjuvants. On a donc recours à de grands principes de formulation. L'optimisation des formules est réalisée par des essais en laboratoire ou, bien souvent, en centrale à béton. Le nombre d'essais à réaliser dépend de la justesse de la formule de départ ; on comprend dès lors le caractère difficile et fastidieux de la formulation des BAP.

\subsection{Objectifs et démarche de l'étude}

L'équation à résoudre lors de la formulation d'un BAP a beaucoup d'inconnues et une solution pas nécessairement unique. La norme française du Béton Prêt à l'Emploi (P18-305) permet certes de fixer certaines inconnues; la masse minimale de ciment est par exemple donnée par la classe d'environnement du béton. L'expérience acquise au cours des dernières années a en outre permis de poser des plages acceptables pour certains paramètres; le rapport gravillon/sable $(\mathrm{G} / \mathrm{S})$ est souvent proche de 1 ; le volume de pâte varie entre 330 et $4001 / \mathrm{m}^{3}$ [AFG 99]. Il existe cependant des méthodes de formulation moins empiriques. On se propose ici de comparer trois approches récurrentes dans la littérature, avec l'objectif d'élargir le champ des possibilités du formulateur. Parmi ces approches, on trouve l'approche japonaise, qui divise le béton en deux parties : les gros granulats et le mortier qui assure la fluidité du béton. Une autre approche consiste à assurer le caractère autoplaçant du béton par la minimisation du volume de la pâte liante. Enfin, une 
approche basée sur l'optimisation du squelette granulaire a été développée au sein du LCPC.

Compte tenu de leurs différences de base, il nous a paru intéressant d'étudier ces trois approches, de formuler des bétons suivant les procédures préconisées et de comparer les propriétés obtenues. Des bétons de différentes granulométries ont ainsi été conçus, sans agent de viscosité, puis confectionnés en laboratoire. Cette recherche s'insérant dans un contrat industriel, la composition des BAP avait pour cadre les trois points suivants. D'abord, les matériaux utilisés sont issus d'une centrale à béton en activité (du groupe VM Matériaux). Ensuite, la formulation s'est faite en respectant la norme P18-305 (environnement 2b1). Enfin, et surtout, le cahier des charges des BAP ne concernait que les propriétés à l'état frais, comme le veut la pratique actuelle. La caractérisation des bétons obtenus a permis d'évaluer l'efficacité et la faisabilité des méthodes sélectionnées. Une étude de l'incidence de la formulation sur les propriétés à l'état durci a été menée par comparaison des BAP avec un béton ordinaire.

\section{Matériaux et procédures expérimentales}

\subsection{Matériaux utilisés}

Les bétons sont fabriqués avec un ciment CEM II/A 42,5 R PM contenant $92 \%$ de clinker et de surface spécifique égale à $3760 \mathrm{~cm}^{2} / \mathrm{g}$. L'addition minérale est un filler calcaire de surface spécifique égale à $3970 \mathrm{~cm}^{2} / \mathrm{g}$ et de répartition granulométrique proche de celle du ciment (figure 1). La défloculation des fines est assurée par l'ajout d'un superplastifiant polycarboxylate polyoxyde, dont la compatibilité avec le ciment a été vérifiée au cône de Marsh [LAR 00].

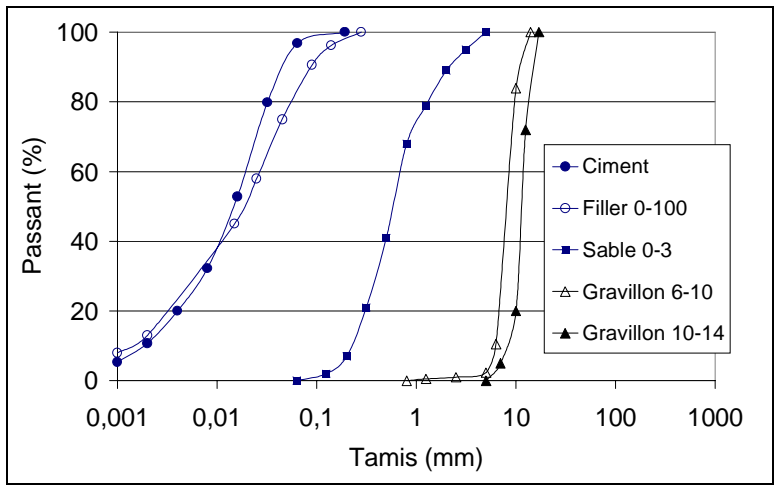

Figure 1. Courbes granulométriques des éléments solides du béton

Le sable est un sable de mer de granulométrie $0 / 3 \mathrm{~mm}$, avec moins de $1 \%$ d'éléments inférieurs à $80 \mu \mathrm{m}$, et comporte quelques éléments coquillés. Les deux 
gravillons utilisés, $6 / 10 \mathrm{~mm}$ et $10 / 14 \mathrm{~mm}$, sont concassés avec forme très anguleuse (aplatissement supérieur à $15 \%$ ). Leurs masses volumiques sont respectivement de $2950 \mathrm{~kg} / \mathrm{m}^{3}$ et $3050 \mathrm{~kg} / \mathrm{m}^{3}$. La figure 1 montre la discontinuité entre le sable et les éléments fins ainsi que l'aspect monogranulaire des gravillons.

\subsection{Caractérisation des bétons à l'état frais}

Les bétons sont fabriqués par gâchée de 40 litres dans un malaxeur Couvrot d'une contenance maximale de 70 litres. La procédure de malaxage est la suivante : malaxage à sec des constituants solides pendant une minute, ajout progressif de l'eau pendant une minute, ajout du superplastifiant, et malaxage du mélange pendant trois minutes. L'ouvrabilité du béton est caractérisée par les essais recommandés par l'Association Française de Génie Civil [AFG 99]. Leur procédure est décrite ci-après.

A la fin du malaxage, dix litres de béton sont versés dans un seau et placés en attente pendant 15 minutes. On réalise pendant ce temps l'essai d'étalement au cône d'Abrams, qui donne une indication sur le seuil de cisaillement du béton et donc sa capacité à «s'autocompacter » en milieu non confiné. Vient ensuite l'essai à la boîte en L (figure 2). La partie verticale de la boîte est remplie de béton; après une minute, la trappe est levée permettant l'écoulement à travers les armatures. Le taux de remplissage final $\mathrm{H} 2 / \mathrm{H} 1$ renseigne sur la mobilité du béton en milieu ferraillé.

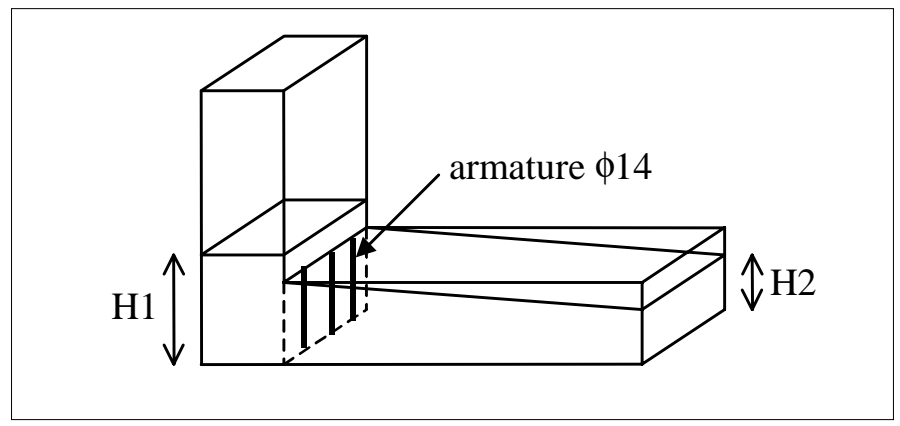

Figure 2. Boîte en $L$

Enfin, on réalise l'essai de stabilité au tamis. Une masse de $4,8 \mathrm{~kg}$ de béton prélevée du seau est versée sur un tamis de maille $5 \mathrm{~mm}$; après deux minutes, on pèse la masse de pâte (laitance) ayant traversé le tamis. Le pourcentage de laitance par rapport à la masse initiale de l'échantillon évalue la résistance à la ségrégation du béton. Le tableau 1 donne les valeurs limites pour chaque essai. Il est à noter que l'essai de mesure du ressuage également préconisé par l'AFGC n'est pas utilisé dans cette étude. 


\begin{tabular}{|l|l|}
\hline Etalement & De 60 à $75 \mathrm{~cm}$ \\
\hline H2/H1 & $\geq 0,8$ \\
\hline Laitance & $\leq 15 \%$ \\
\hline
\end{tabular}

Tableau 1. Cahier des charges d'un BAP préconisé par l'AFGC

\subsection{Mesures des propriétés mécaniques et des déformations libres}

Après gâchage, différentes éprouvettes sont coulées puis conservées dans une ambiance à $20^{\circ} \mathrm{C}$ et $95 \%$ d'humidité relative. Les résistances à la compression et à la traction par fendage sont mesurées sur éprouvettes $11 \times 22 \mathrm{~cm}$ à plusieurs échéances $(1,3,7,14,28$ et 90 jours).

Le module d'élasticité dynamique est déterminé sur éprouvettes 11 x $22 \mathrm{~cm}$ au moyen d'un appareil de mesure non destructif, le Grindosonic, qui permet de mesurer la fréquence d'une vibration créée par un choc léger sur l'éprouvette; le calcul du module est déduit de cette fréquence à partir du modèle développé par Spinner et Tefft [SPI 61].

Des éprouvettes $7 \times 7 \times 28 \mathrm{~cm}$, équipées de plots métalliques à chaque extrémité, sont démoulées à 24 heures puis placées dans une salle climatisée à $20 \pm 1{ }^{\circ} \mathrm{C}$ et $50 \pm 5 \%$ d'humidité relative pour la mesure du retrait total.

Le dispositif schématisé sur la figure 3 est utilisé pour mesurer le retrait plastique, c'est-à-dire les déformations libres dues au séchage avant et pendant la prise. Le béton est coulé juste après la fabrication dans un moule $7 \times 7 \times 28$. Sa surface supérieure est soumise à un séchage à $20{ }^{\circ} \mathrm{C}$ et à $50 \%$ d'humidité relative. Aux extrémités de l'éprouvette, des capteurs «laser» suivent les déformations du béton par l'intermédiaire de deux plaques réfléchissantes. Les mesures commencent trente minutes après le coulage.

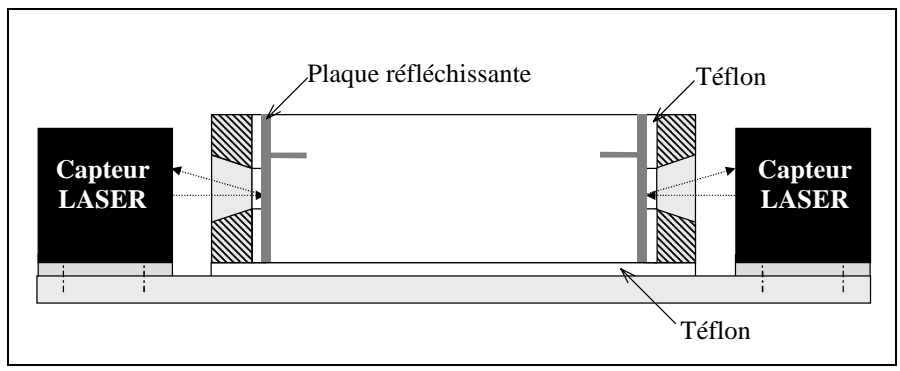

Figure 3. Schéma du dispositif de mesure du retrait plastique 


\section{Différentes approches pour la formulation}

Dans cette partie, sont présentés le principe détaillé des différentes approches et leur application au cas de nos constituants. Pour chaque méthode, nous avons formulé deux bétons différents par la taille du gros granulat : BAP 0/10 et BAP 0/14. La masse de ciment est prise supérieure ou égale à $350 \mathrm{~kg} / \mathrm{m}^{3}$, afin de respecter la norme P18-305.

\subsection{Formulation par la méthode japonaise [OKA 99, OKA 00]}

La formulation des BAP par l'approche développée à l'Université de Kochi se fait de manière sécuritaire, en privilégiant le volume de pâte au détriment des granulats. Les bétons obtenus sont sous-dosés en granulats et par conséquent loin d'un optimum économique. Le surcoût engendré sur le matériau est compensé, au Japon, par les économies sur la main d'œuvre. Les principes de formulation et leur application sont les suivants.

- Les chercheurs japonais ont montré que le risque de blocage est minimisé lorsqu'on limite le volume du gravillon pour $1 \mathrm{~m}^{3}$ à la moitié de sa compacité. Par définition, la compacité d'un mélange de grains est le rapport du volume de grains et du volume total du système grains + vides.

A défaut d'autres indications, nous avons choisi de la mesurer en suivant la procédure du LCPC [SED 00]. La compacité mesurée est pour chaque type de gravillon d'environ 0,57. Dans le cas de la formule BAP 0/10, le volume du gravillon est donc posé à $285 \mathrm{l} / \mathrm{m}^{3}$. Dans le cas de la formule BAP $0 / 14$, ce volume est réparti pour moitié en 6/10 et pour l'autre moitié en 10/14.

- Le volume du sable est posé forfaitairement à $40 \%$ du volume de mortier du béton. La fluidité du béton est garantie par la réduction des frictions granulaires.

- La méthode ne précise pas comment doser le liant ; néanmoins la quantité de ciment peut être fixée, par exemple, en respectant la donnée des normes (soit ici une masse minimale de ciment de $350 \mathrm{~kg} / \mathrm{m}^{3}$ ). Les rapports massiques eau sur ciment et filler sur ciment peuvent également être choisis sur des critères de résistance.

- Les dosages de l'eau et du superplastifiant sont déterminés par des essais sur mortiers dont le volume de sable est fixé à $40 \%$. On réalise des essais d'étalement à l'aide d'un cône à mortier et d'écoulement à l'entonnoir. Les chercheurs japonais ont montré que la relation entre l'étalement et l'écoulement est linéaire lorsque le dosage en eau varie à quantité de superplastifiant constante. A l'aide de peu d'essais, il est alors possible de déterminer un couple adjuvant/eau optimal permettant de fluidifier le mortier.

Lors des essais sur mortiers, nous avons préféré fixer le rapport massique filler sur ciment à 0,8 , et non pas poser la masse de ciment à $350 \mathrm{~kg} / \mathrm{m}^{3}$, ce qui aurait conduit à un volume de filler supérieur à celui du ciment (il existe à l'heure actuelle peu de données dans la littérature sur de telles contenances de filler). Les essais ont 
été réalisés à l'aide d'un entonnoir et d'un cône à mortier, dont les dimensions sont la moitié de celles d'un cône d'Abrams. La figure 4 présente le résultat des essais de recherche du couple «eau/superplastifiant». L'étalement normalisé D optimal (équation [1]) est égal à 10 et le temps d'écoulement relatif T optimal (équation [2]) est égal à 1 .

$$
\begin{aligned}
& \mathrm{D}=\frac{\mathrm{d}^{2}-\mathrm{d}_{0}^{2}}{\mathrm{~d}_{0}^{2}} \text {, où } \mathrm{d}: \text { diamètre de la galette, } \mathrm{d}_{0}: \text { base du cône }(\mathrm{cm}) \\
& \mathrm{T}=\frac{10}{\mathrm{t}}, \text { où } \mathrm{t}: \text { temps d'écoulement }(\mathrm{s})
\end{aligned}
$$

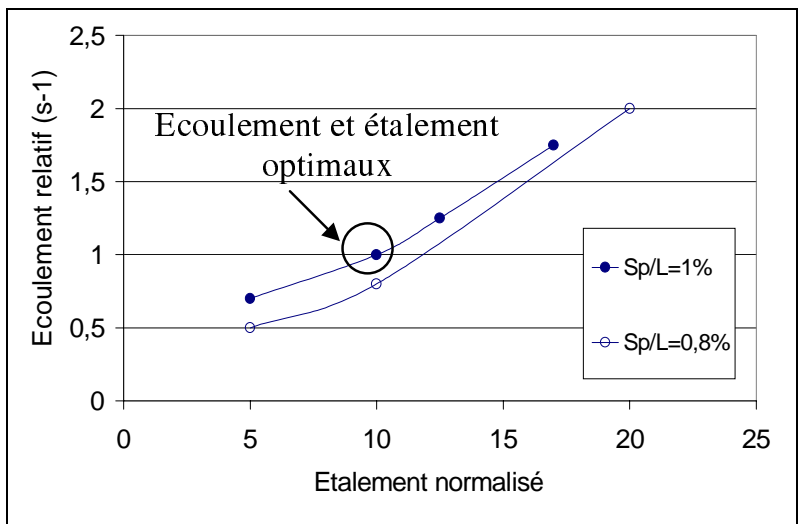

Figure 4. Recherche du dosage en eau et en superplastifiant

Le dosage en superplastifiant trouvé est de $1 \%$ de la masse de fines, le rapport $\mathrm{E} / \mathrm{L}$ de 0,23 et la masse de ciment de $400 \mathrm{~kg} / \mathrm{m}^{3}$. Les formules obtenues sont présentées dans le tableau 4.

\subsection{Formulation par minimisation du volume de pâte [TAN 95, PET 96, VAN 99]}

Le béton est considéré ici comme un mélange biphasique, avec une phase solide (granulats) et une phase liquide (pâte $=$ eau + liant + adjuvants). Les auteurs supposent que la phase liquide joue deux rôles dans un BAP. D'abord, elle fluidifie le matériau en limitant les frottements entre les granulats. Elle écarte ensuite suffisamment les gravillons pour éviter la formation de voûtes contre les armatures, responsables d'un éventuel blocage de l'écoulement. Les auteurs font de plus l'hypothèse qu'il existe un volume minimum de pâte permettant de remplir chacune de ces deux fonctions. Ils proposent les deux critères suivants. 


\subsubsection{Critère de fluidité}

Le volume minimum de pâte nécessaire pour fluidifier $1 \mathrm{~m}^{3}$ de béton s'écrit :

$$
\mathrm{V}_{\mathrm{f} \min \mathrm{i}}=1-\frac{\left(1-\mathrm{V}_{\text {vide }}\right)}{\mathrm{C}_{\mathrm{e}}}
$$

où $\mathrm{V}_{\text {vide }}$ est la porosité $\left(\mathrm{m}^{3} / \mathrm{m}^{3}\right)$ du mélange de sable et de gravillon, mesurée selon la norme américaine ASTM C29/C29M. $C_{e}$ est un coefficient «d'écartement». Lorsqu'il vaut $1, \mathrm{~V}_{\text {fmini }}$ est égal à $\mathrm{V}_{\text {vide }}$ : le volume de pâte remplit la porosité des granulats. Lorsque $\mathrm{C}_{\mathrm{e}}$ est supérieur à 1 , la pâte comble la porosité et écarte les granulats. Ce coefficient est calculé comme suit :

$$
\mathrm{C}_{\mathrm{e}}=\left(\frac{\mathrm{e}_{\min \mathrm{i}}}{\mathrm{D}_{\text {moyen }}}+1\right)^{3} \text { et } \mathrm{D}_{\text {moyen }}=\frac{\sum\left(\mathrm{m}_{\mathrm{i}} \times \mathrm{d}_{\mathrm{i}}\right)}{\sum \mathrm{m}_{\mathrm{i}}}
$$

où $\mathrm{D}_{\text {moyen }}$ est le diamètre moyen des granulats $(\mathrm{mm}) ; \mathrm{d}_{\mathrm{i}}$ est le diamètre du tamis $\mathrm{i}$ $(\mathrm{mm}) ; \mathrm{m}_{\mathrm{i}}$ est la masse de granulats sur le tamis $\mathrm{i}(\mathrm{kg}) ; \mathrm{e}_{\operatorname{mini}}$ est la distance moyenne minimale entre les granulats pour fluidifier le béton $(\mathrm{mm})$.

La détermination de $\mathrm{e}_{\operatorname{mini}}$ se fait de manière expérimentale. Des bétons avec des volumes de pâte décroissants sont confectionnés ; on considère que le volume limite de fluidité est atteint lorsque l'étalement du béton est inférieur à $65 \mathrm{~cm}$ ou lorsqu'il y a ségrégation. Au final, on obtient des courbes donnant la distance $\mathrm{e}_{\min }$ en fonction de $D_{\text {moyen }}$ pour différents rapports massiques eau sur fines $(E /(C+F))$ et pour différents diamètres maximaux du gravillon. On notera que les auteurs n'ont choisi que trois paramètres pour décrire $\mathrm{e}_{\operatorname{mini}}\left(\mathrm{E} /(\mathrm{C}+\mathrm{F}), \mathrm{D}_{\operatorname{moyen}}, \mathrm{D}_{\max }\right)$ au détriment, par exemple, du dosage en superplastifiant. Ils expliquent ce choix par un souci de simplicité du modèle.

\subsubsection{Critère de non-blocage}

$\mathrm{Vb}_{\mathrm{i}}$ représente le volume limite de granulats de taille $\mathrm{d}_{\mathrm{i}}$, au-delà duquel le béton ne s'écoule plus dans un milieu confiné, comme la boîte en L. Les auteurs proposent de modéliser $\mathrm{Vb}_{\mathrm{i}}$ linéairement en fonction du rapport de l'espacement e des armatures de la boîte en $\mathrm{L}$ et du diamètre $\mathrm{d}_{\mathrm{i}}$ (figure 5). On remarque que le volume critique d'un sable quelconque $\left(\mathrm{e} / \mathrm{d}_{\mathrm{i}}>15\right)$ est de $8401 / \mathrm{m}^{3}$ : le sable n'est pas prépondérant dans le critère du blocage, si le béton est fluide. Le reste de la courbe, c'est-à-dire l'ordonnée du point $\mathrm{A}$, est à déterminer expérimentalement pour les gravillons utilisés dans le béton à formuler. Il y a donc un grand nombre d'essais à réaliser pour obtenir la courbe du volume critique. Dans notre étude, nous utilisons les équations de la courbe que les auteurs ont publiées pour un gravillon concassé. 


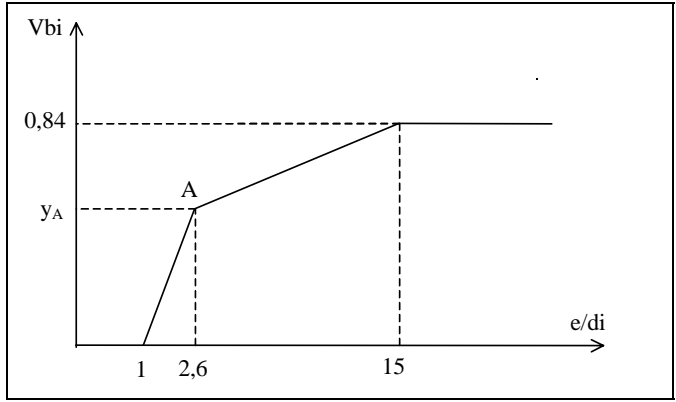

Figure 5. Volume limite de blocage $V b_{i}$ en fonction du rapport de l'espacement des armatures de la boîte en $L$ et le diamètre $d_{i} d u$ granulat

Les auteurs écrivent le risque de blocage en fonction du rapport du volume $V_{i}$ de granulat $\mathrm{d}_{\mathrm{i}}$ et de son volume limite $\mathrm{V}_{\mathrm{bi}}$ :

$$
\mathrm{R}=\sum_{\mathrm{i}} \frac{\mathrm{V}_{\mathrm{i}}}{\mathrm{V}_{\mathrm{bi}}}
$$

En posant $\mathrm{R}=1$, ils en déduisent l'expression [5], qui donne le volume de pâte, pour $1 \mathrm{~m}^{3}$ de béton, en deçà duquel il y a risque de blocage lors de l'écoulement du béton en milieu confiné :

$$
\mathrm{V}_{\mathrm{b} \text { min } \mathrm{i}}=1-\frac{\rho_{\mathrm{g}}+\left(\rho_{\mathrm{s}}-\rho_{\mathrm{g}}\right) \times \mathrm{N}_{\mathrm{g}}}{\sum \frac{\mathrm{p}_{\mathrm{gi}} \times \mathrm{N}_{\mathrm{g}} \times \rho_{\mathrm{s}}}{\mathrm{Vb}_{\mathrm{i}}}+\sum \frac{\mathrm{p}_{\mathrm{si}} \times\left(1-\mathrm{N}_{\mathrm{g}}\right) \times \rho_{\mathrm{g}}}{\mathrm{Vb}_{\mathrm{i}}}}
$$

où $\rho_{\mathrm{g}}$ et $\rho_{\mathrm{s}}$ sont les masses volumiques respectives du gravillon et du sable $\left(\mathrm{kg} / \mathrm{m}^{3}\right)$; $\mathrm{N}_{\mathrm{g}}$ est le rapport entre la masse de gravillon et la masse totale de granulats; $\mathrm{p}_{\mathrm{gi}}$ (respectivement $\mathrm{p}_{\mathrm{si}}$ ) est la proportion dans le gravillon (respectivement dans le sable) du diamètre $\mathrm{d}_{\mathrm{i}}(\%)$.

\subsubsection{Application de la méthode}

Pour le calcul des volumes de pâtes minimaux suivant les deux critères, nous avons utilisé les données publiées par les auteurs, concernant le coefficient $\mathrm{e}_{\operatorname{mini}}$ et la fonction $\mathrm{Vb}_{\mathrm{i}}=\mathrm{f}\left(\mathrm{e} / \mathrm{d}_{\mathrm{i}}\right)$. La figure 6 montre le résultat des calculs dans le cas du béton BAP $0 / 10$ et pour un rapport $\mathrm{E} /(\mathrm{C}+\mathrm{F})$ égal à 0,3 (critère de fluidité). Le critère de non-blocage est largement prépondérant par rapport à celui de la fluidité. On retrouve un résultat similaire dans le cas de la formule BAP 0/14, pour différentes répartitions entre gravillons $6 / 10$ et $10 / 14$, et quel que soit le rapport $E /(C+F)$, compris entre 0,3 et 0,4 . 


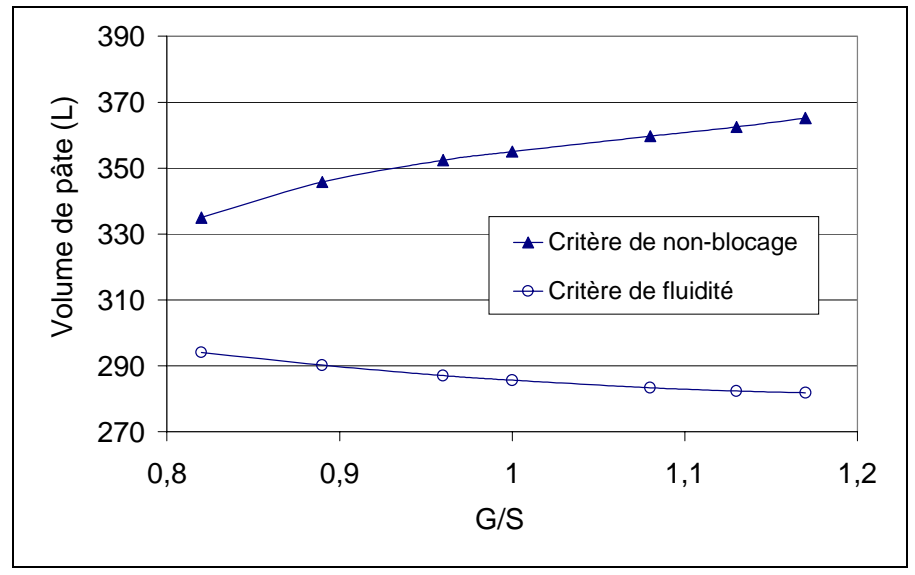

Figure 6. Critère de non-blocage et de fluidité (formule BAP 0/10)

Le critère de non-blocage nous donne un volume de pâte fonction croissante du rapport massique gravillon sur sable $(\mathrm{G} / \mathrm{S})$. Le choix du $\mathrm{G} / \mathrm{S}$ pourrait se faire de manière arbitraire en posant par exemple $G / S=1$. Du point de vue économique, il serait préférable de prendre un rapport $\mathrm{G} / \mathrm{S}$ le plus faible possible pour limiter la quantité de liant. On sait cependant qu'un béton fortement dosé en sable entraîne plus d'air. Nous préférons ici, de manière classique, choisir le mélange sable gravillons avec une porosité minimale. L'optimisation est faite à l'aide du logiciel René-LCPC [SED 00] (tableau 2).

\begin{tabular}{|c|c|c|}
\hline Répartition massique & BAP 0/10 & BAP 0/14 \\
\hline Gravillon 10/14 (\%) & 0 & 41 \\
\hline Gravillon 6/10 (\%) & 53 & 12 \\
\hline Sable (\%) & 47 & 47 \\
\hline G/S & 1,13 & 1,13 \\
\hline
\end{tabular}

Tableau 2. Optimisation de l'empilement sable/gravillon

Le rapport $\mathrm{E} /(\mathrm{F}+\mathrm{C})$ est imposé normalement par le critère de fluidité. Ce n'est bien sûr pas le cas ici. Nous choisissons de le fixer à 0,3 et d'ajuster l'étalement du béton en jouant sur la quantité de superplastifiant.

Les formules obtenues sont présentées dans le tableau 4. 


\subsection{Formulation par optimisation du squelette granulaire [SED 99a, SED 99b, LAR 00]}

\subsubsection{Principe}

La formulation des bétons passe d'ordinaire par une optimisation de la porosité du système formé par les grains solides, du ciment aux gravillons [BAR 96]. Il est connu, par exemple, que la résistance à la compression augmente avec la compacité de la pâte. L'ouvrabilité est, elle aussi, tributaire de l'arrangement du squelette granulaire. Les nombreuses études consacrées aux suspensions de grains solides montrent en effet qu'il existe une relation directe entre la viscosité du mélange et le rapport entre concentration solide $\phi$ et compacité de l'empilement «sec » des grains. Forts de ces constatations, les chercheurs du LCPC ont essayé de modéliser le comportement du béton à l'état frais à partir du calcul de la compacité de son squelette granulaire. Le modèle de calcul (Modèle d'Empilement Compressible) fait intervenir les deux notions décrites ci-après.

- La compacité virtuelle $\gamma$ correspond à la compacité maximale que pourrait prendre l'empilement si tous les grains étaient rangés de manière optimale. Dans un mélange de $n$ classes granulaires, la compacité virtuelle partielle $\gamma_{i}$ des grains de diamètre $\mathrm{d}_{\mathrm{i}} \mathrm{s}$ 'écrit :

$$
\gamma_{i}=\frac{\beta_{i}}{1-\sum_{j=1}^{i-1} y_{j}\left(1-\beta_{i}+b_{i, j} \beta_{i}\left(1-\frac{1}{\beta j}\right)\right)-\sum_{j=1+i}^{n} y_{j}\left(1-a_{i, j} \frac{\beta_{i}}{\beta_{j}}\right)}
$$

où $y_{j}$ est la proportion volumique des grains de diamètre $d_{j}$ dans l'empilement; $\beta_{i}$ est la compacité virtuelle des grains $\mathrm{d}_{\mathrm{i}}$; c'est une caractéristique propre à cette classe, qui est déduite de mesures de compacité sur une plus grande étendue granulaire (dans le cas du béton : un gravillon, un sable ou une poudre). Les coefficients $a_{i, j}$ et $b_{i, j}$ servent à modéliser respectivement les effets de desserrement et les effets de parois d'une classe sur une autre. Leurs expressions en fonction de $\mathrm{a}_{\mathrm{i}, \mathrm{j}}$ et $\mathrm{b}_{\mathrm{i}, \mathrm{j}}$ ont été calées sur de nombreuses données expérimentales.

- En réalité, un empilement ne peut pas atteindre sa compacité virtuelle. Une grandeur représentative du degré de compaction a donc été créée. Cet indice de serrage $\mathrm{K}^{\prime}$ est défini comme la somme des indices de serrage $\mathrm{K}_{\mathrm{i}}{ }_{\mathrm{i}}$ de chaque classe granulaire $d_{i}$ et s'écrit selon l'équation [7].

$$
K^{\prime}=\sum_{i=1}^{n} K_{i}^{\prime}=\sum_{i=1}^{n}\left(\frac{\frac{y_{i}}{\beta_{i}}}{\frac{1}{\phi}-\frac{1}{\gamma_{i}}}\right)
$$


Pour un indice de serrage $\mathrm{K}^{\prime}$ infini, la compacité réelle $\phi$ du mélange de grains est égale à la compacité virtuelle $\gamma$; on montre en outre que $\gamma$ est égale à la borne inférieure des $\gamma_{\mathrm{i}}$. Dans le cas du béton, la porosité du squelette granulaire (1- $\left.\phi\right)$ est égale aux volumes d'eau et d'air pour $1 \mathrm{~m}^{3}$. L'indice de serrage est représentatif de l'énergie qu'il faut fournir au béton pour qu'il se compacte jusqu'à $\phi$.

Le LCPC a développé un rhéomètre à béton (le BTRHEOM), qui a permis de montrer que le comportement rhéologique des bétons peut être estimé par le modèle de Bingham [LAR 98]. La contrainte de cisaillement dans le béton est une fonction linéaire de la vitesse de déformation :

$$
\tau=\tau_{0}+\mu \gamma
$$

où $\tau_{0}$ et $\mu$ sont respectivement le seuil de cisaillement et la viscosité du béton.

Le comportement à l'état frais du béton dépend de l'arrangement de son squelette granulaire. Il est donc intéressant d'écrire ces deux propriétés, intrinsèques au matériau, à l'aide du Modèle d'Empilement Compressible. La viscosité est posée sous la forme suivante :

$$
\mu=\exp \left(\mathrm{A} \times\left(\frac{\phi}{\phi^{*}}-\mathrm{B}\right)\right)
$$

La concentration solide $\phi$ est donnée par la quantité d'eau dans le béton; la compacité $\phi^{*}$ est définie par les auteurs comme la compacité du squelette obtenue pour un indice de serrage $\mathrm{K}^{\prime}$ égal à 9 [7]. Les coefficients $\mathrm{A}$ et $\mathrm{B}$ ont été calés grâce aux résultats d'essais au rhéomètre. L'erreur sur la viscosité est de $46 \mathrm{~Pa} . \mathrm{s}$ en moyenne. On remarquera que la viscosité écrite ainsi n'est pas fonction du dosage en superplastifiant, ce qui est confirmé par les mesures. Dans la pratique, cette relation montre que, pour diminuer la viscosité d'un béton, il faut augmenter la quantité d'eau (diminuer $\phi$ ) ou doser le squelette de telle sorte qu'il soit le plus compact possible à l'état sec (maximiser $\left.\phi^{*}\right)$.

Les auteurs proposent de modéliser le seuil de cisaillement à l'aide des indices de serrage $\mathrm{K}_{\mathrm{i}}^{\prime}$ des classes granulaires [10]. $\mathrm{K}_{\mathrm{c}}^{\prime}, \mathrm{K}_{\mathrm{f}}^{\prime}, \mathrm{K}_{\mathrm{s}}^{\prime}, \mathrm{K}_{\mathrm{S}}^{\prime}, \mathrm{K}_{\mathrm{g}}^{\prime}, \mathrm{K}_{\mathrm{G}}^{\prime}$ sont respectivement les indices de serrage du ciment, du filler, des éléments inférieurs à $80 \mu \mathrm{m} / \mathrm{m}$ du sable, des éléments supérieurs à $80 \mu \mathrm{m} / \mathrm{m}$ du sable, du petit gravillon, du gros gravillon.

$$
\tau_{0}=\exp \left(\mathrm{a}_{0}+\mathrm{a}_{\mathrm{c}} \mathrm{K}_{\mathrm{c}}^{\prime}+\mathrm{a}_{\mathrm{f}} \mathrm{K}_{\mathrm{f}}^{\prime}+\mathrm{a}_{\mathrm{s}} \mathrm{K}_{\mathrm{s}}^{\prime}+\mathrm{a}_{\mathrm{S}} \mathrm{K}_{\mathrm{S}}^{\prime}+\mathrm{a}_{\mathrm{g}} \mathrm{K}_{\mathrm{g}}^{\prime}+\mathrm{a}_{\mathrm{G}} \mathrm{K}_{\mathrm{G}}^{\prime}\right)
$$

Plus l'indice $\mathrm{K}_{\mathrm{i}}$ est élevé, plus les grains de diamètre $\mathrm{d}_{\mathrm{i}}$ sont proches les uns des autres ; donc, plus l'indice $\mathrm{K}_{\mathrm{i}}$ est élevé, plus les frottements entre ces grains sont 
grands. $\mathrm{K}_{\mathrm{i}}$ représente par conséquent la contribution de la classe i au seuil de cisaillement global. Dans l'expression [10], les indices de serrage partiels sont affectés d'un coefficient $a_{i}$ qui est fonction décroissante du diamètre moyen des grains. On traduit ainsi le fait que les frictions entre grains augmentent quand le diamètre moyen diminue, à volume de solide constant. Les coefficients affectés aux éléments fins sont de plus calculés en fonction du dosage en superplastifiant et de son dosage de saturation.

Les auteurs ont remarqué au cours de leurs essais que la stabilité d'un béton et sa capacité à s'écouler en milieu confiné peuvent être estimées par des valeurs limites sur les indices de serrage des éléments fins $\mathrm{K}_{\mathrm{p}}{ }_{\mathrm{p}}$ et des gros gravillons $\mathrm{K}_{\mathrm{G}}{ }_{\mathrm{G}}$.

Ces modèles rhéologiques sont compilés dans un logiciel de formulation des bétons (BétonlabPro2). A partir des caractéristiques des matériaux, le logiciel simule la plupart des propriétés du béton formulé. Le logiciel est également pourvu d'un module d'optimisation qui calcule à partir d'un cahier des charges précis la formule de béton, optimale suivant un critère donné. Le tableau 3 propose le cahier des charges minimal à rentrer pour un BAP.

\begin{tabular}{|c|c|c|c|}
\hline Propriétés & Cahier des charges & BAP 0/10 & BAP 0/14 \\
\hline$\tau_{0}(\mathrm{~Pa})$ & $\leq 500$ & 371 & 305 \\
\hline$\mu($ Pa.s $)$ & $\leq 300$ & 339 & 350 \\
\hline $\mathrm{K}_{\mathrm{p}}^{\prime}$ & $\geq 3,4$ & 3,4 & 3,4 \\
\hline $\mathrm{K}^{\prime}{ }_{\mathrm{G}}$ & $\leq 1,4$ & 1,3 & 1,2 \\
\hline
\end{tabular}

Tableau 3. Cahier des charges d'un BAP et simulations des propriétés rhéologiques des formules BAP 0/10 et BAP 0/14

\subsubsection{Application}

Nous avons d'abord déterminé les propriétés de nos constituants nécessaires à la formulation d'un BAP (caractérisé uniquement par ses propriétés à l'état frais), à savoir : la compacité du sable et des gravillons, la demande en eau du ciment et du filler en présence de superplastifiant, le dosage à saturation du superplastifiant.

En appliquant le cahier des charges précédent, le calcul ne converge pas vers une solution satisfaisante. Certaines propriétés se contredisent; la viscosité diminue principalement par ajout d'eau, alors que $\mathrm{K}_{\mathrm{p}}$, indicateur de résistance à la ségrégation, augmente par réduction du rapport E/L. Néanmoins, en relâchant la contrainte sur la viscosité (maximum autorisé à $350 \mathrm{~Pa}^{-1} \mathrm{~s}^{-1}$ ), la contradiction est levée. Les propriétés simulées des deux formules sont proposées dans le tableau 3.

On trouvera les formules établies par le logiciel dans le tableau 4. 


\section{Résultats de la formulation et des essais à l'état frais}

Le tableau 4 montre les différentes compositions obtenues avec chaque approche. On remarque que toutes les formules comportent un volume de pâte très important (supérieur à $3501 / \mathrm{m}^{3}$ ).

\begin{tabular}{|c|c|c|c|c|c|c|}
\hline \multirow[b]{2}{*}{$\left(\mathrm{kg} / \mathrm{m}^{3}\right)$} & \multicolumn{3}{|c|}{ BAP $0 / 10$} & \multicolumn{3}{|c|}{ BAP $0 / 14$} \\
\hline & $\begin{array}{l}\text { Approche } \\
\text { japonaise }\end{array}$ & $\begin{array}{c}\text { Minim. } \\
\mathrm{V}_{\text {pâte }}\end{array}$ & $\begin{array}{c}\text { Bétonlab } \\
\text { Pro }\end{array}$ & $\begin{array}{l}\text { Approche } \\
\text { japonaise }\end{array}$ & $\begin{array}{c}\text { Minim. } \\
\mathrm{V}_{\text {pâte }}\end{array}$ & $\begin{array}{c}\text { Bétonlab } \\
\text { Pro }\end{array}$ \\
\hline $10 / 14$ & 0 & 0 & 0 & 436 & 721 & 544 \\
\hline $6 / 10$ & 873 & 917 & 808 & 436 & 211 & 279 \\
\hline Sable & 704 & 813 & 810 & 704 & 827 & 806 \\
\hline Ciment & 400 & 350 & 350 & 400 & 350 & 350 \\
\hline Filler & 330 & 210 & 275 & 330 & 220 & 280 \\
\hline Eau eff. & 170 & 170 & 170 & 170 & 170 & 180 \\
\hline Adjuvant & 7,4 & 5,7 & 6,1 & 7,4 & 5,7 & 5,8 \\
\hline Vpâte (1) & 424 & 364 & 388 & 424 & 368 & 400 \\
\hline $\mathrm{G} / \mathrm{S}$ & 1,24 & 1,13 & 1 & 1,24 & 1,13 & 1,02 \\
\hline $\mathrm{V}_{\mathrm{G}} / \mathrm{V}_{\mathrm{S}}$ & 1,08 & 0,97 & 0,86 & 1,08 & 0,97 & 0,85 \\
\hline $\mathrm{E} / \mathrm{L}$ & 0,23 & 0,31 & 0,28 & 0,23 & 0,26 & 0,28 \\
\hline
\end{tabular}

Tableau 4. Formules obtenues avec les différentes approches

Le tableau 5 donne les résultats des essais de caractérisation. Tous les bétons testés présentent un étalement supérieur à $65 \mathrm{~cm}$, soit une grande fluidité. Aucune couronne de laitance ou de mortier n'a été remarquée en périphérie du béton après étalement. Cette absence de ségrégation est confirmée par les résultats de l'essai au tamis (même si la formule «japonaise $0 / 14$ » atteint la limite préconisée). Les deux bétons composés avec la deuxième méthode ont bloqué dans la boîte en L.

\begin{tabular}{|c|c|c|c|c|c|c|c|}
\hline & \multicolumn{3}{|c|}{ BAP 0/10 } & \multicolumn{3}{|c|}{ BAP $0 / 14$} & \multirow[b]{2}{*}{ Cible } \\
\hline & $\begin{array}{l}\text { Appr. } \\
\text { jap. }\end{array}$ & $\begin{array}{c}\text { Minim. } \\
\mathrm{V}_{\text {pâte }}\end{array}$ & $\begin{array}{l}\text { Béton } \\
\text { labPro }\end{array}$ & $\begin{array}{l}\text { Appr. } \\
\text { jap. }\end{array}$ & $\begin{array}{l}\text { Minim. } \\
\mathrm{V}_{\text {pâte }}\end{array}$ & $\begin{array}{l}\text { Béton } \\
\text { labPro }\end{array}$ & \\
\hline $\begin{array}{l}\text { Etalement } \\
(\mathrm{cm})\end{array}$ & 75 & 72 & 68 & 74 & 68 & 70 & $\geq 60$ \\
\hline $\mathrm{H} 1 / \mathrm{H} 2$ & 0,82 & 0,3 & 0,8 & 0,85 & 0,4 & 0,8 & $\geq 0,8$ \\
\hline $\begin{array}{c}\text { Laitance } \\
(\%)\end{array}$ & 13,8 & 12 & 2 & 15 & 2,6 & 3,9 & $\leq 15$ \\
\hline
\end{tabular}

Tableau 5. Résultats des essais de caractérisation 


\section{Analyses}

\subsection{Approche japonaise}

Il est difficilement envisageable d'utiliser cette méthode à la lettre, car les bétons formulés ainsi ne sont pas viables du point de vue économique. Le prix de revient de telles formules est supérieur de 10 à $20 \%$ par rapport à celui des BAP utilisés actuellement en France. Par ailleurs, les essais de l'AFGC (qui ont tous ici été validés) ne mettent pas en évidence le caractère très visqueux, voire collant, des bétons obtenus. Qu'en est-il par exemple de la qualité des parements qu'on obtiendrait avec des bétons contenant plus de 4201 de pâte ?

La démarche présente cependant beaucoup de pistes à explorer. D'abord, la masse de gravillon, fixée en prenant son volume égal à la moitié de la compacité, est proche de la masse de gravillon employée dans les BAP en France (de l'ordre de $850 \mathrm{~kg} / \mathrm{m}^{3}$ ). De plus, la compacité du gravillon est fonction directement de sa forme : un gravillon anguleux ou plat a une compacité plus faible qu'un gravillon arrondi. Or de la forme dépendent, en partie, la fluidité et le risque de blocage du béton. Il est dès lors judicieux de doser le gravillon à partir de sa compacité. En pratique, il est facile d'évaluer grossièrement cette grandeur, par exemple par piquage des gravillons secs dans un cylindre [SED 00].

L'autre intérêt de la méthode réside dans sa manière d'envisager l'optimisation des dosages en eau et en superplastifiant par des essais sur mortiers. Il existe en effet une bonne corrélation entre les comportements à l'état frais du BAP et de son mortier, en particulier lorsque l'on fait varier les masses d'eau et d'adjuvant. C'est un résultat qui est mis en avant dans quelques publications [BILL 99]. La méthode des Mortiers de Bétons Equivalents (MBE) développée récemment lors d'un projet national en France en est une autre illustration [SCH 00]. Par ailleurs, des essais sur mortiers en laboratoire sont beaucoup moins lourds à réaliser que des essais sur bétons, en centrale à béton.

\subsection{Méthode par minimisation du volume de pâte}

Les essais de caractérisation mettent en évidence le manque de fiabilité du volume de pâte calculé par la méthode : l'essai d'écoulement dans la boîte en L n'a été validé pour aucune des deux compositions. Certes, la loi $\mathrm{V}_{\mathrm{bi}}=\mathrm{f}\left(\mathrm{e} / \mathrm{d}_{\mathrm{i}}\right)$, donnant le volume de gravillon limite, est issue de données de la littérature, donc calée pour des gravillons différents de ceux utilisés dans notre étude. Il est vrai également que, pour appliquer correctement la méthode, il faudrait déterminer expérimentalement les différents coefficients, nécessaires aux deux critères, sur des bétons fabriqués avec nos constituants. En ce sens, la méthode n'est pas concurrentielle par rapport aux approches empiriques actuelles, basées sur de (nombreux) essais d'optimisation des formules. 
Il n'empêche que c'est une approche intéressante de la formulation : formuler un BAP à partir de son volume de pâte. En effet, lorsque le formulateur a réussi à obtenir un béton très fluide, c'est-à-dire un étalement supérieur à $65 \mathrm{~cm}$, la quantité de pâte du béton est le principal levier sur lequel il peut jouer pour répondre aux exigences de l'essai à la boîte en L. La figure 7 montre à ce propos la bonne corrélation entre le volume de pâte et le résultat de l'essai à la boîte en L de nos bétons.

En outre, le critère de non-blocage est facilement programmable dans tout tableur. Il est d'ailleurs possible d'introduire un paramètre de calage permettant de modifier la loi $\mathrm{V}_{\mathrm{bi}}=\mathrm{f}\left(\mathrm{e} / \mathrm{d}_{\mathrm{i}}\right)$, puisqu'elle ne dépend que d'un seul paramètre, l'ordonnée du point A (figure 5).

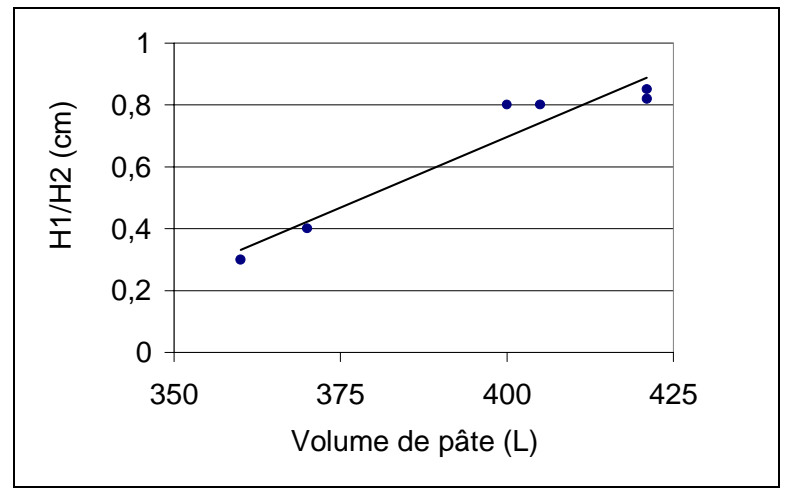

Figure 7. Corrélation entre le taux de remplissage et le volume de pâte des bétons testés, toutes granulométries confondues

\subsection{Approche par optimisation du squelette granulaire}

Le logiciel BétonlabPro2 donne les résultats les plus satisfaisants des trois méthodes, du point de vue à la fois technique et économique. Les formules obtenues sont-elles pour autant optimales? La question se pose en fait face aux masses de fines importantes trouvées par le calcul (plus de $620 \mathrm{~kg} / \mathrm{m}^{3}$ ).

Il est à noter dans un premier temps que les matériaux utilisés dans notre étude sont désavantageux pour la formulation d'un BAP. Les gravillons, en particulier, sont très denses et anguleux; la demande en pâte est donc grande pour stabiliser le béton et le faire s'écouler sans blocage. Il faut ensuite reconnaître que la précision du calcul dépend de la justesse des mesures des propriétés des constituants. Si les mesures de compacité sont reconnues comme fiables, il n'en est pas de même de la détermination du dosage à saturation du superplastifiant. Elle se fait en effet sur pâte, ou même sur mortier, avec un rapport E/L fixé, différent de celui du béton. 
Enfin, la formule calculée est le résultat d'un cahier des charges indicatif. Dans le but d'évaluer ce dernier, les formules obtenues avec les trois méthodes ont été simulées à l'aide du logiciel. Les simulations font ressortir la bonne concordance entre seuil de cisaillement calculé et étalement mesuré (figure 8), et entre indice de non-blocage $\mathrm{K}_{\mathrm{G}}{ }_{\mathrm{G}}$ et essai à la boîte en $\mathrm{L}$ (tableau 6). En revanche, aucune corrélation n'a été trouvée entre l'indice de ségrégation $\mathrm{K}_{\mathrm{p}}^{\prime}$ et le pourcentage de laitance récoltée lors des essais de stabilité au tamis (figure 9). Les formules «japonaises» par exemple ont les indices $\mathrm{K}_{\mathrm{p}}$ les plus élevés, donc sont théoriquement les plus stables, alors qu'elles présentent les plus forts pourcentages de laitance. On peut néanmoins s'interroger sur la pertinence de l'essai de stabilité au tamis pour des bétons contenant plus de 4001 de pâte. L'absence de corrélation entre simulations et mesures trouve peut-être son explication dans le fait que la valeur limite de l'indice $\mathrm{K}_{\mathrm{p}}^{\prime}$ a été déterminée par une mesure directe de la ségrégation sur éprouvette $16 \times 32$ fendue après durcissement, et non par le biais de l'essai au tamis. Il n'en demeure pas moins que cette valeur limite semble sévère.

\begin{tabular}{|c|c|c|c|c|c|c|c|}
\cline { 2 - 7 } \multicolumn{1}{c|}{} & \multicolumn{3}{c|}{ BAP 0/10 } & \multicolumn{3}{c|}{ BAP 0/14 } & \multirow{2}{*}{} \\
\cline { 2 - 7 } \multicolumn{1}{c|}{} & $\begin{array}{c}\text { Appr. } \\
\text { jap. }\end{array}$ & $\begin{array}{c}\text { Minim. } \\
\mathrm{V}_{\text {pâte }}\end{array}$ & $\begin{array}{c}\text { Bétonlab } \\
\text { Pro }\end{array}$ & $\begin{array}{c}\text { Appr. } \\
\text { jap. }\end{array}$ & $\begin{array}{c}\text { Minim. } \\
\mathrm{V}_{\text {pâte }}\end{array}$ & $\begin{array}{c}\text { Bétonlab } \\
\text { Pro }\end{array}$ & Cible \\
\hline $\mathbf{K}_{\mathbf{p}}^{\prime}$ & 4,8 & 3,2 & 3,4 & 5 & 3,3 & 3,4 & $\geq 3,4$ \\
\hline $\begin{array}{c}\text { Laitance } \\
(\boldsymbol{\%})\end{array}$ & 15 & 2,6 & 2 & 13,8 & 12 & 3,9 & $\leq 15$ \\
\hline $\mathbf{K}_{\mathbf{G}}^{\prime}$ & 1,2 & 1,2 & 1,3 & 1,4 & 1,6 & 1,2 & $\leq 1,4$ \\
\hline $\mathbf{H 2} / \mathbf{H} \mathbf{1}$ & 0,82 & 0,4 & 0,8 & 0,85 & 0,3 & 0,8 & $\geq 0,8$ \\
\hline
\end{tabular}

Tableau 6. Simulations des propriétés des six formules

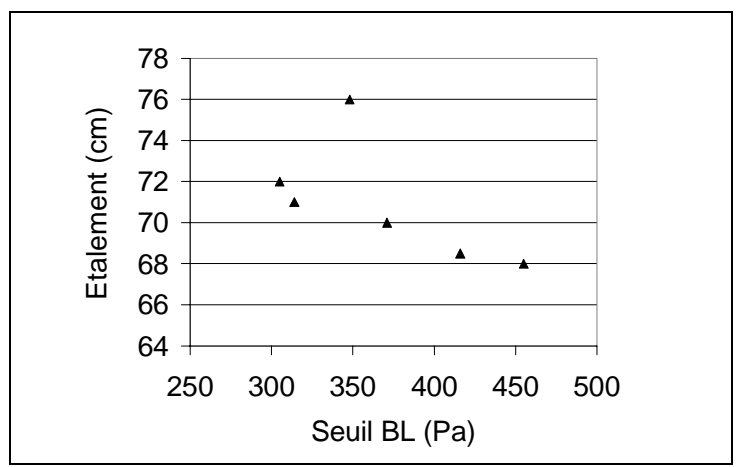

Figure 8. Corrélation entre le seuil de cisaillement calculé par BétonlabPro2 et l'étalement 


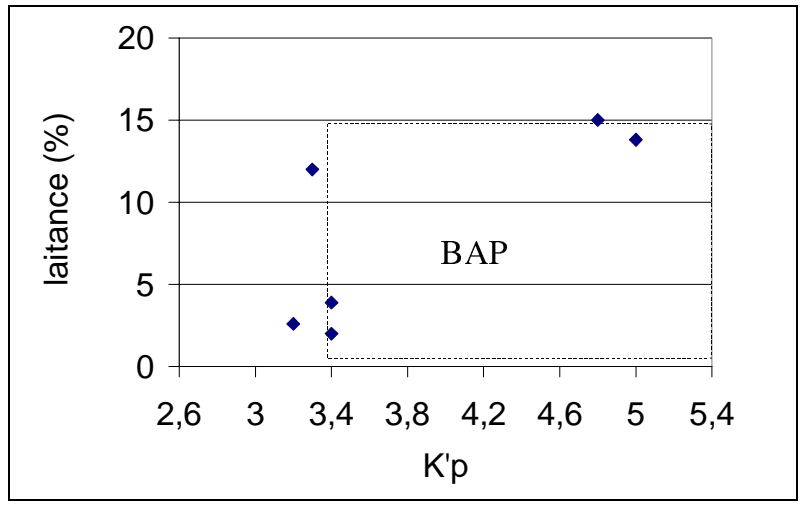

Figure 9. Corrélation entre l'indice de serrage $K_{p}^{\prime}$ calculé par BétonlabPro2 et le pourcentage de laitance mesuré lors de l'essai de stabilité au tamis

\section{Caractérisation des bétons à l'état durci}

On présente ici l'évolution des propriétés mécaniques des bétons de granulométrie 0/10 ayant passé l'obstacle des essais AFGC (formules 0/10 «japonaise » et $0 / 10$ «Bétonlab»). A titre de comparaison, un béton ordinaire de consistance plastique (affaissement de $14,5 \mathrm{~cm}$ ) a été confectionné avec les mêmes matériaux (tableau 7).

\begin{tabular}{|l|c|}
\hline Formule BO & $\mathrm{kg} / \mathrm{m}^{3}$ \\
\hline Gravillon 10/14 & 940 \\
\hline Gravillon 6/10 & 195 \\
\hline Sable & 800 \\
\hline Ciment & 390 \\
\hline Eau efficace & 170 \\
\hline Superplastifiant & 0,65 \\
\hline
\end{tabular}

Tableau 7. Formule du béton ordinaire

La figure 10 présente les résistances en compression (fcj) obtenues par écrasement de trois éprouvettes cylindriques 11 x $22 \mathrm{~cm}$ (écart type : $2 \mathrm{MPa}$ ). Les $\mathrm{BAP}$ ont des résistances élevées, et pratiquement égales, ce que justifient leurs rapports $\mathrm{E} / \mathrm{C}$ très proches. Il est plus surprenant de constater que la résistance à 28 jours des BAP est d'au moins $40 \%$ supérieure à celle du béton ordinaire malgré un rapport E/C voisin. Cela met en évidence le rôle du volume de pâte et du filler 
calcaire sur la compacité du squelette solide des BAP. Ces deux paramètres de formulation ne semblent cependant pas influer sur la cinétique d'hydratation du ciment puisque l'évolution du rapport $\mathrm{fcj} / \mathrm{fc} 28$ est identique pour les trois bétons (figure 11).

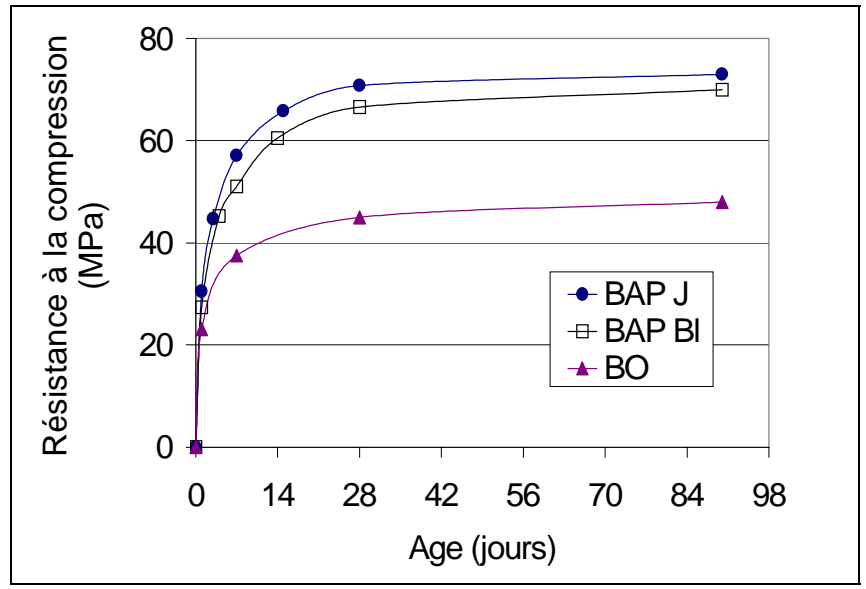

Figure 10. Evolution de la résistance à la compression

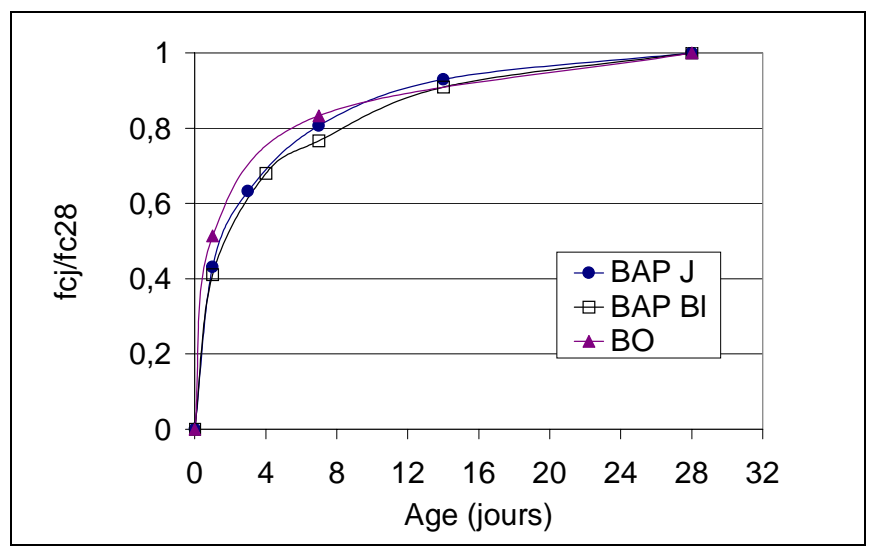

Figure 11. Evolution du rapport $f c j / f c 28$

L'augmentation des résistances en compression des BAP s'accompagne d'une augmentation de la résistance en traction (ft), mesurée par fendage (figure 12). A 28 jours, le rapport $\mathrm{ft} / \mathrm{fc}$ des BAP est pratiquement du même ordre que celui $\mathrm{du}$ béton ordinaire, soit $1 / 12$. 


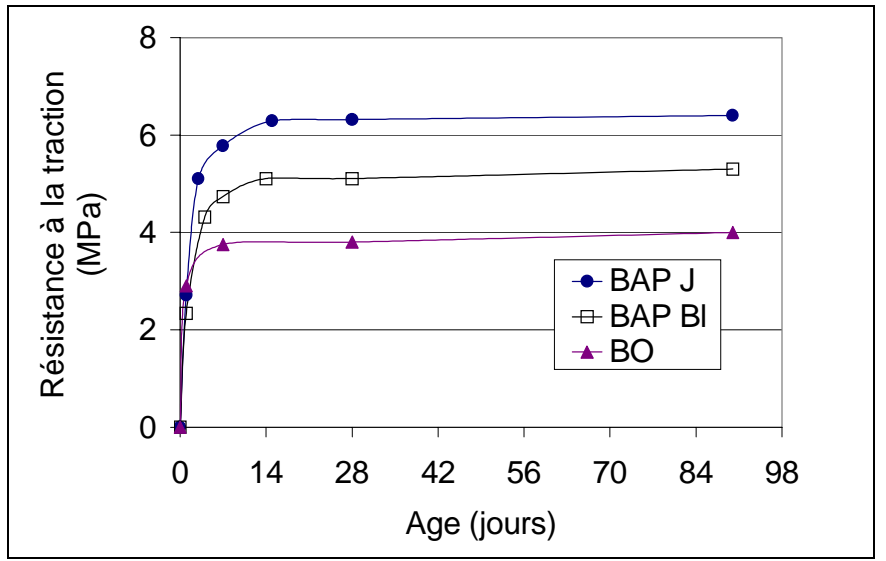

Figure 12. Evolution de la résistance à la traction

L'autre propriété importante des bétons durcis est le module d'élasticité. Dans cette étude, nous avons suivi l'évolution du module à l'aide du Grindosonic, qui donne un module dynamique (figure 13). Malgré une résistance plus grande, les BAP ont un module inférieur ou égal à celui du béton ordinaire. Les trois bétons ayant été confectionnés avec les mêmes granulats, cette différence est due au volume de pâte plus élevé pour les BAP. On peut penser qu'à résistances égales, un BAP, formulé avec une forte quantité de pâte, sera plus souple qu'un béton ordinaire. D'autres études sur le module des BAP, couplées à la quantification de l'énergie de fissuration, sont nécessaires pour statuer sur la question.

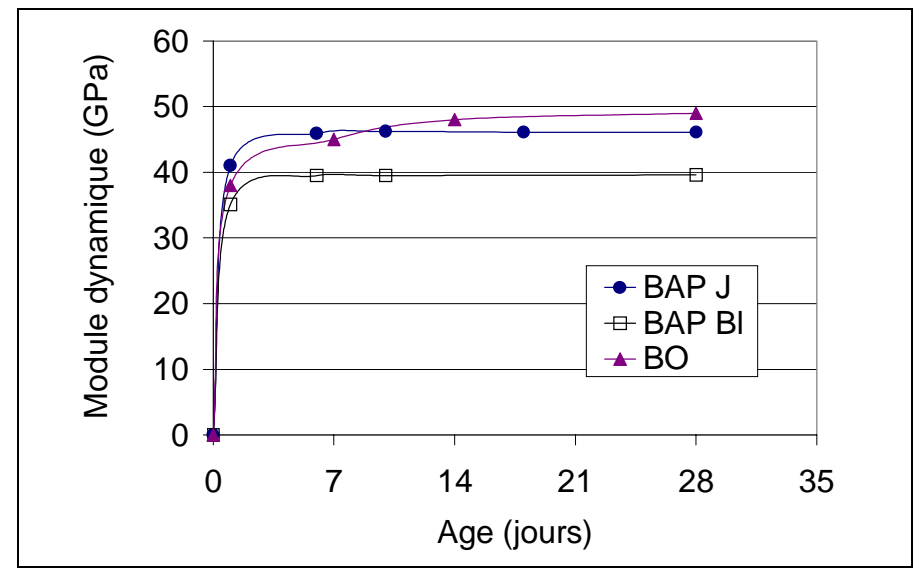

Figure 13. Evolution du module dynamique 


\section{Déformations libres}

Par définition, nous appelons retrait plastique la déformation libre du béton avant la fin de prise. C'est la conséquence du retrait d'autodessiccation, dû aux réactions d'hydratation, et du retrait lié au séchage à la surface du béton. Sa mesure n'est pas standardisée et dépend du type de dispositif utilisé : volumique ou linéique [BAR 99]. Si l'essai volumique est facilement réalisable sur pâte ou sur mortier [LOU 00], il est en revanche délicat sur béton, à cause des granulats et du risque de ressuage [JUS 93]. De plus, il ne permet pas d'apprécier l'effet du séchage sur l'amplitude du retrait plastique. Pour cette étude, nous avons donc choisi de quantifier le retrait plastique des bétons avec le dispositif présenté sur la figure 3, qui a l'avantage pratique de mesurer les déformations dès la naissance d'une cohésion. On montre ainsi que les deux BAP ont un fort retrait avant et pendant la prise, comparé à celui du béton ordinaire (figure 14). Leur importante quantité de fines, associée à un faible rapport eau sur fines, est à l'origine de cette différence [RAD 94]. La grande amplitude du retrait plastique des BAP est également favorisée par le retard de prise, engendré par le superplastifiant. Des études plus approfondies sont actuellement en cours pour mieux identifier les phénomènes physiques responsables de ces déformations et sur les moyens pour les limiter. Pour l'heure, une cure efficace est à prévoir dès que le béton autoplaçant est susceptible de sécher pendant sa prise (dalles, tabliers, etc.), d'autant que le risque de fissuration semble accru pour un retrait plastique dépassant $1 \mathrm{~mm} / \mathrm{m}$ [HOL 00].

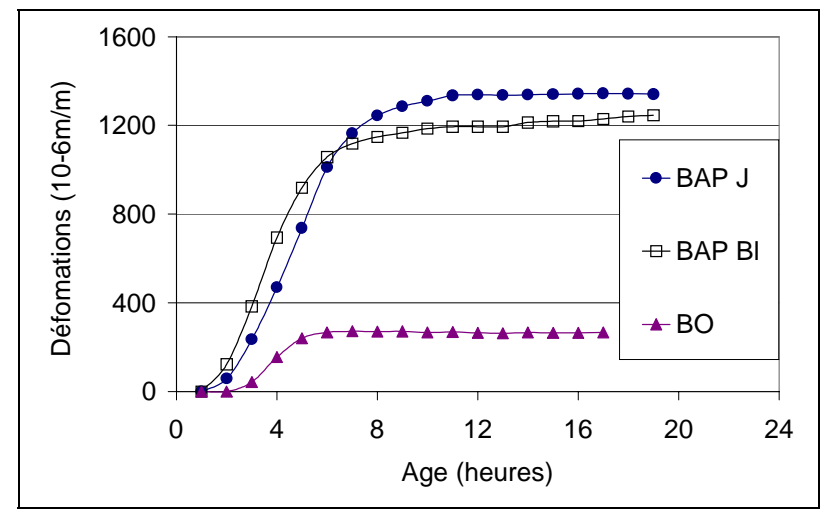

Figure 14. Evolution du retrait plastique

Les mesures du retrait endogène ont débuté après 24 heures de mûrissement. La figure 15 montre que l'amplitude de ce retrait, conséquence de l'hydratation du ciment, est faible pour les trois bétons testés (inférieure à $150 \mu \mathrm{m} / \mathrm{m}$ à 3 mois). Cela est lié à leur rapport $\mathrm{E} / \mathrm{C}$ élevé, qui conduit à la formation d'un réseau poreux composé en majorité de pores capillaires, où les tensions capillaires restent faibles. 
Les bétons présentent des cinétiques et des amplitudes de retrait total sensiblement identiques jusqu'à 100 jours (figure 16). On en déduit que leurs retraits de dessiccation sont également les mêmes. Ce résultat peut paraître étonnant puisque les bétons ont des volumes de pâte très différents. Il contredit en cela une étude récente qui montre que l'amplitude du retrait de dessiccation est directement proportionnelle au volume de pâte [BIS 99]. Cependant, puisque leurs rapports E/L sont plus faibles, le squelette des BAP est plus compact que celui du BO, ce que confirment les essais de compression. Leur réseau poreux étant moins ouvert sur l'extérieur, les transferts d'humidité sont par conséquent plus réduits. On peut donc penser que la différence de porosité entre BAP et BO compense leur différence de volume de pâte, vis-à-vis du séchage.

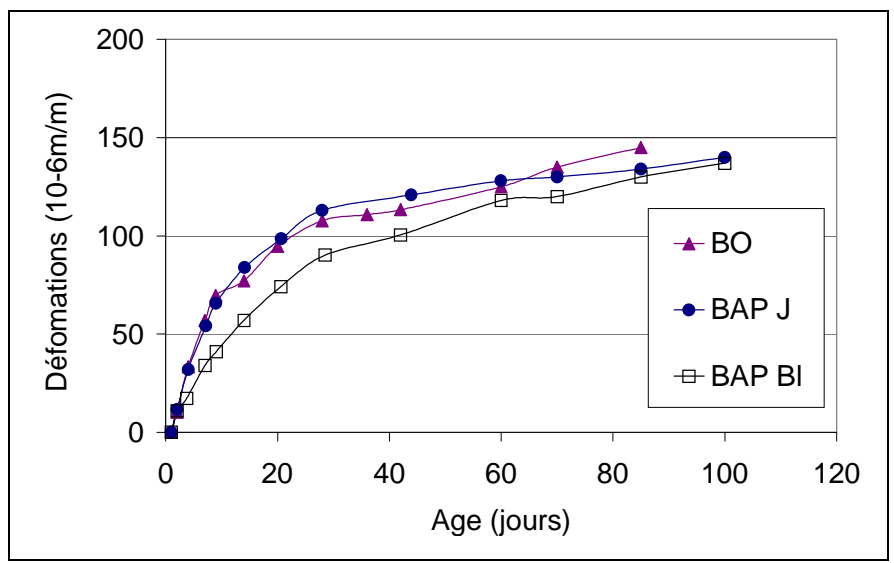

Figure 15. Evolution du retrait endogène

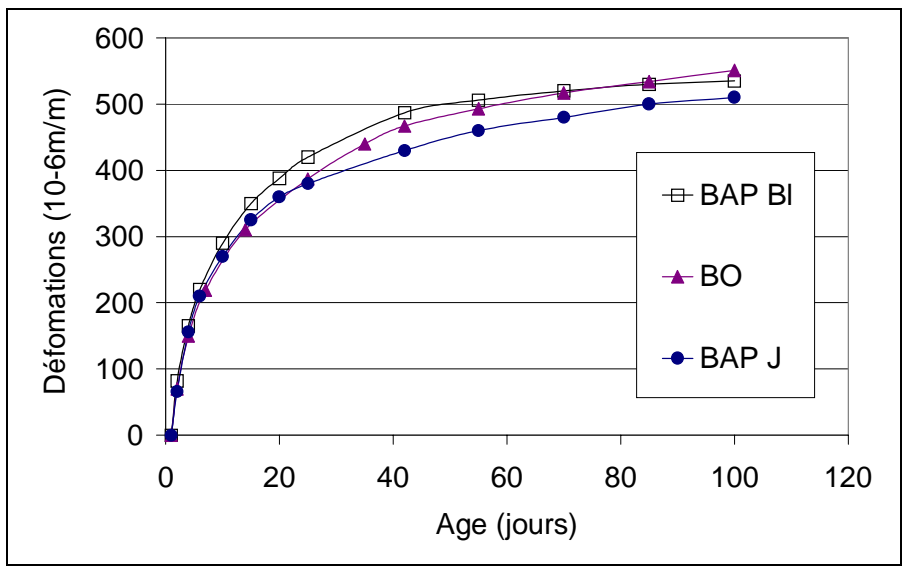

Figure 16. Evolution du retrait total 


\section{Conclusions}

\subsection{Formulation}

Cette étude a permis de faire le point sur trois méthodes de formulation des BAP et de comparer expérimentalement les caractéristiques rhéologiques obtenues. Bien que les résultats soient issus d'un nombre limité d'essais, nous pouvons d'ores et déjà dresser les conclusions suivantes :

- toutes les méthodes testées demandent un certain nombre d'essais pour caractériser les matériaux ou leurs interactions. Une mesure de la compacité des granulats est nécessaire dans chaque méthode. Les essais en laboratoire sont donc pratiquement indispensables à une formulation scientifique des BAP ;

- le béton formulé par l'approche japonaise est très visqueux et renferme un volume de pâte trop important. Déduire le volume de gravillons d'une mesure de la compacité peut cependant être un élément intéressant pour rationaliser l'approche actuelle de la formulation ;

- étant donné le grand nombre d'équations à résoudre, l'utilisation de moyens informatiques ne semble pas superflue pour formuler un BAP. La programmation dans un tableur du critère de non-blocage de la méthode de minimisation de la pâte peut être un premier pas d'une informatisation de la formulation ;

- le logiciel Bétonlab Pro2 est certainement l'outil informatique, et pédagogique, le plus performant à l'heure actuelle pour la composition des BAP, puisque au moyen de simulations il permet d'appréhender l'influence de chaque constituant sur le comportement du béton. Néanmoins, si une bonne corrélation a été trouvée entre l'étalement et le seuil de cisaillement calculé, cela n'a pas été le cas entre l'indice de ségrégation et les résultats des essais au tamis. La valeur limite de non-ségrégation proposée dans Bétonlab Pro 2 est, d'après nos essais, sévère ;

- dans chaque méthode, quelques inconnues subsistent, par exemple le rapport addition/ciment. Le choix devra se faire sur des critères mécaniques, économiques ou de durabilité.

Le tableau 8 récapitule d'un point de vue pratique les principales différences entre les trois approches. Envisagée de cette manière, la composition des BAP présente un besoin d'équipements, informatiques ou de laboratoire, a priori absents chez la plupart des producteurs de bétons prêts à l'emploi ; une telle rationalisation de la formulation doit s'accompagner d'une acquisition de nouveau matériel.

La mise au point d'une formule théorique n'est à elle seule pas suffisante. Quelle que soit la méthode utilisée, la formule du béton doit être aussi testée, si possible en centrale à béton, à l'aide des essais de l'AFGC. Il manque à ce jour un document officiel proposant des techniques d'ajustement d'une formule de BAP, comme en avait proposées T. Sedran dans sa thèse [SED 99]. 


\begin{tabular}{|c|c|c|c|}
\hline & Approche japonaise & $\begin{array}{l}\text { Minimisation de Vpâte } \\
\text { (Critère de non-blocage) }\end{array}$ & $\begin{array}{c}\text { Optimisation du } \\
\text { squelette granulaire } \\
\text { (Bétonlab Pro) }\end{array}$ \\
\hline $\begin{array}{l}\text { Inconnues } \\
\text { à fixer } \\
\text { a priori }\end{array}$ & $\begin{array}{l}\text { - Répartition } \\
\text { entre les gravillons } \\
\text { - Masse de ciment } \\
\text { - (Volume de sable) }\end{array}$ & $\begin{array}{l}\text { - Répartition } \\
\text { entre les gravillons, G/S } \\
\text { - Masse de ciment } \\
\text { - Eau et adjuvant }\end{array}$ & - (Masse de ciment) \\
\hline $\begin{array}{l}\text { Essais } \\
\text { en amont }\end{array}$ & $\begin{array}{l}\text { - Essais sur mortier } \\
\text { - Compacités }\end{array}$ & - Aucun & $\begin{array}{l}\text { - Caractérisation des } \\
\text { matériaux }\end{array}$ \\
\hline $\begin{array}{l}\text { Essais } \\
\text { en aval }\end{array}$ & $\begin{array}{l}\text { - Vérification } \\
\text { de la formule }\end{array}$ & $\begin{array}{l}\text { - Ajustement } \\
\text { de la formule (dosage } \\
\text { du superplastifiant...) }\end{array}$ & $\begin{array}{l}\text { - Vérification } \\
\text { de la formule }\end{array}$ \\
\hline $\begin{array}{l}\text { Matériel } \\
\text { nécessaire }\end{array}$ & $\begin{array}{l}\text { - Malaxeur à mortier } \\
\text { - Cylindre 16x32 } \\
\text { (pour la mesure } \\
\text { de la compacité) }\end{array}$ & $-\mathrm{PC}$ & $\begin{array}{l}\text { - Bétonlab Pro } \\
\text { - Malaxeur à mortier } \\
\text { - Mesure compacités } \\
\text { - Cône de Marsh } \\
\text { - PC }\end{array}$ \\
\hline
\end{tabular}

Tableau 8. Comparaison pratique des différentes approches de formulation

\subsection{Comparaison BAP / Béton ordinaire}

Les bétons autoplaçants testés dans cette étude ont des volumes de pâte élevés (supérieurs à $385 \mathrm{l} / \mathrm{m}^{3}$ ). Le rapport entre leur résistance à la compression et leur module d'élasticité a donc été logiquement trouvé supérieur à celui du béton ordinaire. Malgré leur différence de volume de pâte, bétons autoplaçants et béton ordinaire présentent un même retrait total. En terme de durabilité, ces résultats peuvent se traduire par une sensibilité des bétons autoplaçants vis-à-vis de la fissuration due à un empêchement des déformations libres au plus égale à celle du béton ordinaire. Une étude plus complète, avec des essais de retrait empêché, doit être menée pour confirmer cette idée.

\section{Bibliographie}

[AFG 00] AFGC, Les bétons autoplaçants : recommandations provisoires, Ed. AFGC, 2000.

[BAR 96] BARON J., OlLIVIER J.P., Les bétons, bases et données pour leur formulation, Eyrolles, 1996, p. 187-398. 
[BAR 99] BARCElo L., Rigaud S., ACKer P., Clavaud B., "Linear vs volumetric autogenous shrinkage measurement : Material behaviour or experimental artefact?", Proceedings of Self-desiccation and its importance in concrete tecnology, Lund, 1999, p. 109-125.

[BIL 99] BILlBEG P., Fine mortar rheology in mix design of SCC, RILEM PRO7, p. 321-332, 1999.

[BIS 99] Bissonnette B., Pierre P., Pigeon M., "Influence of key parameters on drying shrinkage of cementitious materials", Cement and Concrete Research, 29, 1999, p. 1655-1662.

[HOL 00] Holt E., MARKKU L., "Methods of reducing early age shrinkage", Proceedings of the International RILEM Workshop Shrinkage 2000, RILEM PRO17, 2000, p. 435-447.

[JUS 94] Justnes H., Reyniers B., VAn Loo D., Sellevold E.J., "An Evaluation of Methods for Measuring Chemical Shrinkage of Cementitious Paste", Nordic Concrete Research, Publication 14, 1994, p. 45-61.

[LAR 98] De Larrard F., Ferraris C.F., «Rhéologie du béton frais remanié », Bull. des laboratoires des Ponts et Chaussées, 213, janvier-février, 1998, p. 73-89.

[LAR 00] De LARRARD F., Structures granulaires et formulation des bétons, Ouvrage du Laboratoire Central des Ponts et Chaussées, 2000, p. 249-254.

[LOU 00] Loukili A., Chopin D., Khelidj A., Le Touzo J-Y., “A new approach to determine autogenous shrinkage of mortar at early age considering temperature history", Cement and Concrete Research, 30 (6), 2000, p. 915-922.

[OKA 99] OKAmuRA H., OUCHI M., Self-compacting concrete, Development, present use and future, RILEM PRO7, 1999, p. 3-14.

[OKA 00] OKAMURA H., Ozawa K., OUCHI M., "Self-compacting concrete”, Structural Concrete, $\mathrm{n}^{\circ}$ 1, March, 2000, p. 3-17.

[PET 96] Petersson P., Billberg P., Van B.K., "A model for Self-compacting concrete", Production Methods and Workability of Concrete, édité par Bartos P.J.M., Marrs D.L., Cleland D.J., publié par E\&FN Spon, 1996.

[RAD 94] RADOCEA A., "A model of plastic shrinkage", Magazine of Concrete Research, vol. 46, $\mathrm{n}^{\circ} 167,1994$, p. 125-132, juin.

[SCH 00] Schwartzentruber A., Catherine C., «La méthode du mortier de béton équivalent (MBE) : un nouvel outil d'aide à la formulation des bétons adjuvantés », Materials and Structures, vol. 33, $\mathrm{n}^{\circ} 232,2000$, p. 475-482.

[SED 99a] SEDRAN T., De LARRARD F., Optimization of self-compacting concrete thanks to packing model, RILEM PRO7, 1999, p. 321-332.

[SED 99b] SEDRAN T., Rhéologie et rhéométrie des bétons. Applications aux bétons autonivellants, Thèse du LCPC, 1999.

[SED 00] Sedran T., De Larrard F., Manuel d'utilisation de René-LCPC, Logiciel d'optimisation granulaire, version 6.1d, 2000. 
[SPI 61] SPINNER E., TEFFT W.E., "A method for determining mechanical resonace frequencies and for calculating elastic moduli from these frequencies", Proceeding ASTM, vol. 61 .

[TAN 95] TANGTERMSIRIKUL S., VAN B.K., "Blocking criteria for aggregate phase of selfcompacting high performance concrete", Proceedings of regional symposium on infrastructure development in civil engineering, 19-20 décembre, 1995, p. 58-69.

[VAN 99] VAN B.K., MONTGOMERY D., Mixture porportioning method for self-compacting high performance concrete with minimum paste volume, RILEM PRO7, 1999, p. 373-384. 